\title{
ORIGINAL ARTICLE International Spinal Cord Injury Quality of Life Basic Data Set
}

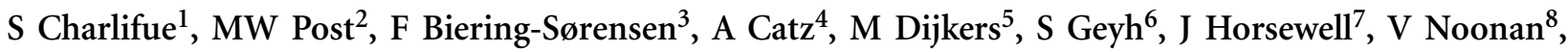 \\ L Noreau $^{9}$, D Tate $^{10}$ and KA Sinnott ${ }^{11}$
}

Study design: Survey of expert opinion, feedback, and development of final consensus.

Objective: Present the background, purpose, development process and results for the International Spinal Cord Injury (SCI) Quality of Life (QoL) Basic Data Set.

Setting: International.

Methods: A committee of experts was established to select and define data elements to be included in an International SCI QoL Basic Data Set. A draft data set was developed and disseminated to appropriate organizations for comment. All suggested revisions were considered, and a final version of the QoL data set was completed.

Results: The QoL data set consists of 3 variables: ratings of satisfaction with general quality of life, satisfaction with physical health, and satisfaction with psychological health. All variables are rated on a scale ranging from 0 (completely dissatisfied) to 10 (completely satisfied).

Conclusion: Collection of the International SCI QoL Basic Data Set variables should be a part of all future studies of SCl QoL to facilitate comparison of results across published studies from around the world. Additional standardized instruments to assess other aspects of QoL can be administered based on the purpose of a particular study.

Spinal Cord (2012) 50, 672-675; doi:10.1038/sc.2012.27; published online 27 March 2012

Keywords: spinal cord injury; quality of life; international data set

\section{INTRODUCTION}

Following the onset of spinal cord injury (SCI) or development of a spinal cord lesion caused by a disease process (SCI/D), an individual is likely to experience varying degrees of body impairment. Such impairments are liable to be permanent and can change a person's life significantly, because of the psychological impact of the loss of body function, and because they may result in activity limitations and participation restrictions. As a result, perceived quality of life $(\mathrm{QoL})$ is likely to change, usually for the worse. Many researchers have investigated QoL following SCI/D, but there has been variation in study design, inclusion criteria, and measures used. ${ }^{1}$ More disagreement exists on the definition and measurement of QoL than on any other construct that plays a critical role in medical, rehabilitative, social and psychological research. ${ }^{2}$ There is as yet no single definition of QoL on which everyone agrees, in spite of plentiful efforts directed at clarifying or defining this concept. QoL has been defined to include both subjective appraisals of one's situation as well as objective and observable aspects, such as limitations imposed on an individual by environmental barriers. ${ }^{3}$ Three approaches to operationalize and measure QoL are suggested by the body of literature, including (1) health related quality of life (HRQoL), (2) well-being, and (3) QoL as a superordinate construct. ${ }^{4}$
HRQoL describes difficulties in functioning caused by poor mental or physical health, ${ }^{5}$ whereas well-being (or subjective well-being) ${ }^{6}$ is commonly described as consisting of three components: positive affect, negative affect, and life satisfaction. The superordinate construct of QoL includes both HRQoL and well-being.

The evaluative or subjective approach defines QoL as a judgment on and reaction to the (in)congruence between aspirations (wishes, desires, goals, plans, etc.) and accomplishments (possessions, statuses, roles, etc.) as perceived by the person involved. Research in this vein may explore life satisfaction, feelings of well being, morale and happiness. In the social sciences, subjective QoL (or well-being) is generally thought to consist of a cognitive and an affective part. The cognitive part is the evaluation of one's life, mostly measured as the satisfaction with one's life as a whole or with particular domains. The affective part consists of both positive (joy, positive mood) and negative emotions (depressive feelings, anxiety, etc). ${ }^{7}$ Following this approach, subjective QoL can be defined as 'reflecting an individual's overall perception of and satisfaction with how things are in their life.'

Clearly QoL, even if defined simply as well-being, is a multifaceted concept. Because the concept involves reactions to the circumstances of life, studies have explored relationships of QoL with many factors

${ }^{1}$ Craig Hospital, Englewood, CO, USA; ${ }^{2}$ Rehabilitation Centre De Hoogstraat and Rudolf Magnus Institute for Neuroscience, Utrecht, The Netherlands; ${ }^{3}$ Clinic for Spinal Cord Injuries, Neuroscience Centre, Rigshospitalet, and Faculty of Health Sciences, University of Copenhagen, Copenhagen, Denmark; ${ }^{4}$ Loewenstein Rehabilitation Hospital, Raanana, and Tel-Aviv University, Tel-Aviv, Israel; ${ }^{5}$ Mt Sinai School of Medicine, New York, NY, USA; ${ }^{6}$ Swiss Paraplegic Research, Nottwil, Switzerland; ${ }^{7}$ The European Spinal Cord Injury Federation, Copenhagen, Denmark; ${ }^{8}$ University of British Columbia, Vancouver, British Columbia, Canada; ${ }^{9}$ Université Laval and Center for Interdisciplinary Research in Rehabilitation and Social Integration (CIRRIS), Quebec, Canada; ${ }^{10}$ University of Michigan, Ann Arbor, MI, USA and ${ }^{11}$ University of Otago, Christchurch, New Zealand Correspondence: Dr S Charlifue, Research, Craig Hospital, 3425 South Clarkson Street, Englewood, CO 80113 USA. E-mail: Susie@craighospital.org

Received 12 December 2011; revised 24 January 2012; accepted 18 February 2012; published online 27 March 2012 
inside and outside the person, including social support, personality, perceived physical health and psychological health, to name a few. Assessing these determinants may be useful in order to capture a general picture of an individual's QoL. In addition, it is important to recognize the effect of cultural factors in shaping QoL. The World Health Organization addressed this issue in their WHOQOL project, defining QoL as 'an individual's perception of his/her position in life in the context of the culture and value systems in which he/she lives, and in relation to his/her goals, expectations, standards and concerns.' ${ }^{\text {A }}$ Although not explicitly stated in this WHO definition, the environment is yet another factor influencing perceptions of QoL-as may be evident comparing the life of someone with SCI in a fully accessible urban environment vs. in an agricultural setting.

\section{Measurement issues}

By definition, a rating of subjective QoL can only be provided by the person him or herself. Such self-ratings are often mistrusted as being inherently unstable and invalid. Although there certainly are problems with self-ratings, specific approaches to questions may minimize these issues. For example, the danger of reflecting only momentary moods that might change quickly may be reduced by asking people to describe how they have felt 'during the past few weeks', instead of how they feel 'now'.

The selection of appropriate QoL measures needs to take into account the psychometric properties of these measures as well as their content and purpose. There are many ways of ensuring that measures are appropriate and have strong psychometric properties. For example, response bias caused by reluctance to express negative emotions or dissatisfaction to others can be reduced by using selfadministered measures and by using a series of indirect questions instead of one question with an obvious target. Measurement error can be reduced by using multi-item questionnaires, although single items perform quite satisfactorily. ${ }^{10}$ Research has shown that responses to measures of happiness, mood, and other aspects of well-being usually correlate strongly with each other, indicating substantial concurrent validity. Non-response and 'don't know' responses tend to be infrequent. Within-person variability of life satisfaction ratings is much lower than variability in mood. ${ }^{11}$ Most people claim to be satisfied, and perceive themselves as happier than average, and it is quite possible that most people truly are satisfied with life, and that we underestimate satisfaction of others because misery is more salient than prosperity. ${ }^{10}$

To address the many different ways QoL can be measured while reducing measurement error and maintaining consistency in these evaluations, the International SCI QoL Basic Data Set was developed. This data set is part of the International SCI Data Sets project which is an effort by the International Spinal Cord Society (ISCoS) and the American Spinal Injury Association (ASIA) to create internationally recognized and endorsed standard data sets relevant to SCI that clinicians and researchers can use in their practice and investigations. This data set development process will allow data to be easily combined and compared to improve our understanding of SCI, its sequelae and natural history, as well as the impact of various interventions. Basic data sets are intended for use in clinical settings as well as research; to date, a core data set has been published, ${ }^{12}$ as well as International SCI Basic Data Sets for lower urinary tract function, ${ }^{13}$ urodynamics, ${ }^{14}$ urinary tract imaging, ${ }^{15}$ bowel function, ${ }^{16}$ female sexual and reproductive function, ${ }^{17}$ male sexual function, ${ }^{18}$ pain, ${ }^{19}$ cardiovascular function, ${ }^{20}$ and metabolic and endocrine function. ${ }^{21}$ Extended data sets are intended to be used in research and to date include data sets for bowel function ${ }^{22}$ and external causes of injury. ${ }^{23}$

Thus, the purpose of the International SCI QoL Basic Data Set is to standardize the collection and reporting of a minimal amount of information necessary to merge and compare results of published and unpublished studies on QoL. Like all basic data sets, it was designed to include a minimal number of data elements, which together can be collected in routine clinical practice. For the purposes of this data set, and to ensure its international applicability and comparability, we use the definition of QoL provided by Wood-Dauphinée et al. ${ }^{8}$ The International SCI QoL Basic Data Set therefore reflects subjective QoL and not other definitions of QoL. Thus, each person is asked to assess his or her own QoL from a personal perspective and utilize his or her own internal standards and evaluations to do so. In this assessment, people may consider all factors that they feel contribute to their QoL, whether these are related to health, finances, family or other factors.

\section{METHODS}

The first draft of the International SCI QoL Basic Data Set was created by a working group consisting of members selected by the group's chair (Charlifue) based on their expertise in the field of QoL research, together with representatives of the Executive Committee of the International SCI Standards and Data Sets (Biering-Sørensen, Post, Noonan). The development process for the International SCI QoL Basic Data Set involved the following steps:

(1) The working group developed a first draft data set during a 3-day meeting in Copenhagen in October, 2008. This data set was further refined with frequent e-mail communication among the group members and subsequently included development of a syllabus for the data set.

(2) The draft data set was reviewed by members of the Executive Committee of the International SCI Standards and Data Sets project.

(3) Comments from the Committee members were discussed among the working group via e-mail and appropriate changes were made to the data set.

(4) Members of the ISCoS Scientific Committee and the ASIA Board of Directors were asked to review the data set.

(5) Comments from the Committee/Board members were discussed among the working group via e-mail and further adjustments were made to the data set.

(6) Relevant and interested international scientific professional organizations and societies as well as interested individuals were invited to review the data set. In addition, the data set was posted on both the ISCoS and ASIA websites for 3 months with a request for comments and suggestions. Comments were received and reviewed by the working group: it was determined that based on feedback, no further changes were necessary.

(7) The data set was finalized May of 2009, having undergone essentially no changes from the originally proposed draft.

(8) Standard naming conventions for the variables of the data set were completed in collaboration with the National Institute of Neurological Diseases and Stroke (NINDS)/National Institutes of Health (NIH) Common Data Element (CDE) project in the United States and KAI Research, Inc. This process is described elsewhere. ${ }^{24}$

(9) Endorsement of the final data set will be sought from additional relevant international organizations and societies.

The International SCI QoL Basic Data Set is applicable to adult individuals with traumatic or non-traumatic spinal cord lesions. To ensure that data are collected in a uniform and consistent manner, each variable has been specifically defined. Use of a standard format is essential for combining data from multiple investigators and locations. Because of the relative simplicity of the International SCI QoL Basic Data Set, it is recommended that the proposed format is adopted. Additional questions can be added at the discretion of the clinicians or investigators if they wish to explore other factors related to QoL. The use of standardized instruments assessing subjective well-being such as the 
Diener Satisfaction with Life Scale, ${ }^{25}$ measures of depressive symptomatology, stress, or other instruments to assess other constructs can be at their discretion.

\section{RESULTS}

The complete data set form is included in the Appendix. The data syllabus and data sheet are available on the ISCoS website (www.iscos.org.uk).

\section{Date of data collection}

As the collection of QoL data may be carried out at any time following SCI/D, the date of data collection is needed to compute time since injury and to identify the data collected in relation to other data collected on the same individual at various time points (that is, using the International SCI Core Data Set). ${ }^{12}$ The working group recommends administering the questions from the International SCI QoL Basic Data Set only after an individual has left the acute setting and is in inpatient rehabilitation or has been discharged to a community setting.

\section{Satisfaction with general quality of life (overall well-being)}

This variable is a numeric self-rating that documents how the individual with a SCI/D subjectively rates his or her general QoL in the past four weeks on a $0-10$ scale, with $0=$ completely dissatisfied and $10=$ completely satisfied. While a consistent definition of 'general QoL' would be ideal, the working group (supported by the literature) determined that no single definition fits all individuals in all settings. Therefore, each individual being queried should consider what factors contribute to his or her QoL and what standards she or he has for QoL, and answer within that context.

\section{Satisfaction with physical health}

This variable is a numeric self-rating that documents how the individual with a SCI/D rates his or her satisfaction with his or her physical health in the past four weeks on a $0-10$ scale, with $0=$ completely dissatisfied and $10=$ completely satisfied .

\section{Satisfaction with psychological health}

This variable is a numeric self-rating that documents how the individual with a SCI/D rates her or his satisfaction with her or his psychological health, emotions and mood in the past four weeks on a $0-10$ scale, with $0=$ completely dissatisfied and $10=$ completely satisfied.

\section{DISCUSSION}

To keep this data set as useful as possible and for ease of administration, we have kept the number of variables as small as possible. The working group came to consensus regarding the 3 items, which are felt to address the most relevant components of subjective QoL. It is recommended that the International SCI Core Data Set ${ }^{12}$ be completed and used in conjunction with the International SCI QoL Basic Data Set. The Core Data Set includes information regarding date of birth, date of injury/onset, gender, etiology of lesion and neurologic status. These variables are necessary to facilitate any analyses, for instance correlating QoL with various demographic or injury/lesion-related factors.

The QoL data set has not yet undergone psychometric testing, but its use is recommended based on experience with other, similar instruments that request rating subjective states or experiences (for example, life satisfaction, pain) on a $0-10$ numeric rating scale. It provides clinicians and researchers information regarding an individual's satisfaction with their general, physical and emotional health, and when used over time, can track changes in those perceptions. However, psychometric criteria like, for example, test-retest reliability apply and should be examined in future research. This brief data set further could be used as a screening measure to identify individuals with SCI/D with possible adjustment problems, for whom targeted assessments with well-validated measures is indicated. An Extended SCI QoL Data Set, to be developed, might provide guidance on standardized measures of choice.

Administration of the International SCI QoL Basic Data Set to individuals with SCI/D whose primary language is not English will require translation of the various components. The working group recommends that a careful translation process be used that follows the published guidelines for translating the International SCI Data Sets. ${ }^{26}$ While linguistic equivalence may be attained, further research has to indicate to what degree there is functional equivalence in various languages.

Having a standard way to consistently assess QoL across different settings, cultures and environments will allow comparison of research results and clinical data worldwide. While there may remain differences in interpretation of QoL concepts, the International SCI QoL Basic Data Set is a significant step toward unifying our ability to record and report this important information.

Although the International SCI QoL Basic Data Set has been reviewed by the international community, it is anticipated that the data set will require periodic revisions based on findings from studies utilizing the Data Set. Ideas for improvement of the Data Set are welcome and should be forwarded to the corresponding author.

\section{DATA ARCHIVING}

There were no data to deposit.

\section{CONFLICT OF INTEREST}

The authors declare no conflict of interest.

\section{ACKNOWLEDGEMENTS}

Coloplast A/S, Denmark, supported the work on this data set with an unconditional grant. We are thankful for comments and suggestions received from Michael DeVivo, Jenny Moses and Eric Weerts. The names of the organizations and societies, which endorse the International SCI QoL Basic Data Set will be posted on the ISCoS website (www.iscos.org.uk)

1 Hill MR, Noonan VK, Sakakibara BM, Miller WC. Quality of life instruments and definitions in individuals with spinal cord injury: a systematic review. Spinal Cord 2010; 48: 438-450.

2 Dijkers MP. Quality of life of individuals with spinal cord injury: a review of conceptualization, measurement, and research findings. J Rehabil Res Dev 2005; 42(Suppl 1): 87-110.

3 Whiteneck GG. The 44th Annual John Stanley Coulter Lecture. Measuring what matters: key rehabilitation outcomes. Arch Phys Med Rehabil 1994; 75: 1073-6.

4 Post M, Noreau L. Quality of life after spinal cord injury. J Neurol Phys Ther 2005; 29 139-146.

5 Geyh S, Fellinghauer BA, Kirchberger I, Post MW. Cross-cultural validity of four quality of life scales in persons with spinal cord injury. Health Qual Life Outcomes 2010; 8: 94.

6 Diener E, Suh EM, Lucas RE, Smith HL. Subjective well-being: three decades of progress. Psychol Bull 1999; 125: 276-302.

7 Argyle M. The Psychology of Happiness, 2nd edn Routledge: London, 2001.

8 Wood-Dauphinee S, Exner G, Bostanci B, Glass C, Jochheim KA, Kluger P et al. Quality of life in patients with spinal cord injury-basic issues, assessment, and recommendations. Restor Neurol Neurosci 2002; 20: 135-149.

9 World Health Organization. Study protocol for the World Health Organization project to develop a Quality of Life assessment instrument (WHOQOL). Qual Life Res 1993; 2 153-19.

10 Veenhoven R. Developments in satisfaction research. Soc Indic Res 1996; 37: 1-46.

11 Eid M, Diener E. Global judgments of subjective well-being: situational variability and long-term stability. Soc Indic Res 2004; 65: 245-277. 
12 DeVivo M, Biering-Sorensen F, Charlifue S, Noonan V, Post M, Stripling T et al. International spinal cord injury core data set. Spinal Cord 2006; 44: 535-540.

13 Biering-Sorensen F, Craggs M, Kennelly M, Schick E, Wyndaele JJ. International lower urinary tract function basic spinal cord injury data set. Spinal Cord 2008; 46 325-330.

14 Biering-Sorensen F, Craggs M, Kennelly M, Schick E, Wyndaele JJ. International urodynamic basic spinal cord injury data set. Spinal Cord 2008; 46: 513-516.

15 Biering-Sorensen F, Craggs M, Kennelly M, Schick E, Wyndaele JJ. International urinary tract imaging basic spinal cord injury data set. Spinal Cord 2009; 47 379-383.

16 Krogh K, Perkash I, Stiens SA, Biering-Sorensen F. International bowel function basic spinal cord injury data set. Spinal Cord 2009; 47: 230-234.

17 Alexander MS, Biering-Sorensen F, Elliott S, Kreuter M, Sonksen J. International spinal cord injury female sexual and reproductive function basic data set. Spinal Cord 2011; 49: 787-790.

18 Alexander MS, Biering-Sorensen F, Elliott S, Kreuter M, Sonksen J. International spinal cord injury male sexual function basic data set. Spinal Cord 2011; 49: 795-798.

19 Widerstrom-Noga E, Biering-Sorensen F, Bryce T, Cardenas DD, Finnerup NB, Jensen MP et al. The international spinal cord injury pain basic data set. Spinal Cord 2008; 46: 818-823.
20 Krassioukov A, Alexander MS, Karlsson AK, Donovan W, Mathias CJ, Biering-Sorensen F. International spinal cord injury cardiovascular function basic data set. Spinal Cord 2010; 48: 586-590.

21 Bauman WA, Biering-Sorensen F, Krassioukov A. The international spinal cord injury endocrine and metabolic function basic data set. Spinal Cord 2011; 49: 1068-1072.

22 Krogh K, Perkash I, Stiens SA, Biering-Sorensen F. International bowel function extended spinal cord injury data set. Spinal Cord 2009; 47: 235-241.

23 Lee BB, Cripps RA, Woodman RJ, Biering-Sorensen F, Wing P, Campbell R et al. Development of an international spinal injury prevention module: application of the international classification of external cause of injury to spinal cord injury. Spinal Cord 2010; 48: 498-503.

24 Biering-Sorensen F, Charlifue S, Devivo MJ, Grinnon ST, Kleitman N, Lu Y et al. Incorporation of the international spinal cord injury data set elements into the National Institute of Neurological Disorders and Stroke Common Data Elements. Spinal Cord 2011; 49: 60-64.

25 Diener E, Emmons RA, Larsen RJ, Griffin S. The satisfaction with life scale. J Pers Assess 1985; 49: 71-75.

26 Biering-Sorensen F, Alexander MS, Burns S, Charlifue S, DeVivo M, Dietz V et al. Recommendations for translation and reliability testing of international spinal cord injury data sets. Spinal Cord 2011; 49: 357-360.

\section{APPENDIX}

INTERNATIONAL SPINAL CORD INJURY DATA SETS

QUALITY OF LIFE BASIC DATA SET - DATA FORM (Version 1.0)

Date performed: (YYYYMMDD) Unknown

1. Thinking about your own life and personal circumstances, how satisfied are you with your life as a whole in the past four weeks? Please use a scale ranging from $\mathbf{0}$ (completely dissatisfied) to 10 (completely satisfied). You can use 0 or 10 or any number in between.

Completely dissatisfied Completely satisfied $\square 0 \quad \square 1 \quad \square 2 \quad \square 3 \quad \square 4 \quad \square 5 \quad \square 6 \quad \square 7 \quad \square 8 \quad \square 9 \quad \square 10$

2. How satisfied are you with your physical health in the past four weeks? Please use a scale ranging from 0 (completely dissatisfied) to 10 (completely satisfied). You can use 0 or 10 or any number in between.

Completely dissatisfied

Completely satisfied

\section{$\square 0 \quad \square 1 \quad \square 2 \quad \square 3 \quad \square 4 \quad \square 5 \quad \square 6 \quad \square 7 \quad \square 8 \quad \square 9 \quad \square 10$}

3. How satisfied are you with your psychological health, emotions and mood in the past four weeks? Please use a scale ranging from 0 (completely dissatisfied) to $\mathbf{1 0}$ (completely satisfied). You can use $\mathbf{0}$ or 10 or any number in between.

Completely dissatisfied

Completely satisfied

$\begin{array}{lllllllllll}\square & \square 1 & \square 2 & \square 3 & \square 4 & \square 5 & \square 6 & \square 7 & \square 8 & \square 9 & \square 10\end{array}$ 